\title{
ERRATUM
}

Ziyou Xiong

\section{Audio-visual sports highlights extraction using Coupled Hidden Markov Models}

Published online: 22 December 2005

(C) Springer-Verlag London Limited 2005

\section{Pattern Anal Appl (2005) 8:62-71}

Due to an unfortunate error, the three coauthors were omitted from this article. Their names and addresses are listed below:

Regunathan Radhakrishnan

Mitsubishi Electric Research Laboratories,

Cambridge, MA, USA.

E-mail: regu@merl.com

Ajay Divakaran

Mitsubishi Electric Research Laboratories,

Cambridge, MA, USA

E-mail: ajayd@merl.com

Thomas S. Huang

Department of Electrical and Computer Engineering, University of Illinois at Urbana-Champaign,

Urbana, IL, USA

E-mail: huang@ifp.uiuc.edu

The original article can be found at http://dx.doi.org/10.1007/ s10044-005-0244-7

\section{Z. Xiong}

Department of Electrical and Computer Engineering,

University of Illinois at Urbana-Champaign, Urbana, IL 61801,

USA

E-mail: zxiong@ifp.uiuc.edu 